\title{
IMPROVEMENT OF THE SIGNAL TO NOISE RATIO BY MEANS OF WAVELET MODULATION
}

\author{
MEJORA DE LA SEÑAL DE RUIDO \\ MEDIANTE MODULACION WAVELET
}

Laércio Alves Nogueira ${ }^{1}$, José Roberto Amazonas².

\begin{abstract}
${ }^{1}$ Student Member, IEEE, Escola Politécnica of the University of São Paulo. laercio@lcs.poli.usp.br.
${ }^{2}$ Member, IEEE, Escola Politécnica of the University of São Paulo. jra@lcs.poli.usp.br.
\end{abstract}

\section{RESUMEN}

En un canal multiusuario (1) donde las señales son corrompidas por ruído blanco aditivo gaussiano (AWGN) e interferencia de acceso múltiple (MAI), un acceso múltiple por división de código (CDMA) receptor detecta los símbolos, o bits, y las estimaciones de los parámetros: un la transformada wavelet se aplica a los símbolos recibidos, que han sido codificados previamente en el transmisor por un código especial, el resultado final es una reducción del ruido (eliminación de ruido), lo que significa la mejora del rendimiento y de la tasa de error de bit del sistema .

Palabras claves: Wavelets, modulacion, eliminación de ruido

\begin{abstract}
In a multiuser channel (1) where signals are corrupted by Additive White Gaussian Noise (AWGN) and Multiple Access Interference (MAI), a code-division multiple access (CDMA) receiver detects the symbols, or bits, and the parameters estimates: a wavelet transform is applied to the received symbols, which have been previously coded at the transmitter by a special code; the final result is a reduction of the noise (denoising), which means improvement of the performance and of the bit error rate of the system.
\end{abstract}

Keywords: Wavelets, modulation, denoising. 


\section{INTRODUCTION}

The Bit Error Rate - BER - of any transmission system is a decreasing function of the Signal to Noise Ratio SNR. So, in order to improve the BER, it is necessary to increase the SNR. However, practical systems are power limited, and the signal power can be increased only up to a certain value. On the other hand, the noise arises from physical reasons and cannot be reduced to any desired value. To overcome such limitations, different processing techniques are employed both at the transmission side and at reception side as well to improve the system's performance. One of the possible techniques to reduce the noise at the receiving side employs the wavelet transform. The wavelet transform is a representation in the plane time-frequency. Any signal can be completed recovered by means of the wavelet coefficients and the inverse wavelet transform $(2,3)$.

An approximation to a typical real signal, in a communications system, can be obtained using a subset of the wavelet coefficients, which form a basis for the mathematical set, or space, under consideration. If, for example, the received signal has a reasonable SNR but not good enough to achieve the desired SNR, the elimination of the wavelet coefficients that are less than a given threshold may result in an improved SNR because the small coefficients carry more information about the noise than the desired signal. On the other hand, there are certain signals that have the energy concentrated in a part of the spectrum. So, if only the wavelet coefficients corresponding to this part of the spectrum are used, the SNR also improves. However, in general, in digital transmission systems none of aforementioned conditions prevails.

\section{MATERIALS AND METHODS}

Consider the information sequence represented by $\{b(m)\}$, in which for the case of Binary Phase Shift Keying - BPSK modulation, the value of each information bit can be \pm 1 . Let's also consider the transmission of a data block of length $N$. Then $\{b(m)\}=\{b(1), b(2), \ldots$, $b(N)\}$. The wavelet spectrum of such signals, unless for some special cases, is similar to the wavelet spectrum of white noise, i.e., the energy is uniformly spread along the whole frequency range. Taking into account also the fact that in real systems the SNR at the receiving side can be very small, even less than 1 , then none of the appropriate conditions for the elimination of some wavelet coefficients from the signal's representation is present.

The Continuous Wavelet Transform - CWT - of a signal $x(t)$ consists of a set $C=\left\{W_{\psi}(s, \tau), s \in \mathfrak{R}^{+}, \tau \in \mathfrak{R}\right\}$, in which

$\begin{array}{ll}- & \tau \text { is the time localization parameter; } \\ \text { - } & \quad \mathfrak{R} \text { is the real set; } \\ \text { - } & s \text { represents scale; and } \\ \text { - } & \psi \quad \psi \text { denotes a wavelet function, }\end{array}$

of wavelet coefficients on the continuous time-scale plane (also known as time-frequency plane) given by

$$
\begin{gathered}
W_{\psi}(s, \tau)=\left\langle\psi_{0_{(s, \tau)}}, x\right\rangle=\int_{-\infty}^{\infty} \frac{1}{\sqrt{s}} \psi_{0}^{*}\left(\frac{\lambda-\tau}{s}\right) x(\lambda) d \lambda, \\
\psi_{0_{(s, \tau)}}(t)=s^{-1 / 2} \psi_{0}\left(\frac{t-\tau}{s}\right)
\end{gathered}
$$

Denotes a dilated and shifted version of "mother" wavelet $\psi_{0}(t)$.

The Discrete Wavelet Transform - DWT - coefficients for continuous time signals can be directly from (4)

$$
\begin{aligned}
\omega_{j, k} & =\left\langle\psi_{\left.0_{\left(2^{j}, 2^{j} k\right.}\right)}, x\right\rangle \\
& =\int_{-\infty}^{\infty} 2^{-j / 2} \psi_{0}^{*}\left(2^{-j} \lambda-k\right) x(\lambda) d \lambda
\end{aligned},
$$

in which the indices $\mathrm{j}$ and $\mathrm{k}$ are called scale and localization, respectively, it does not involve any discrete-time signal, but the continuous-time signal $x(t)$. Equation [2] can also be used with discrete-time signals.

Consider now that the original signal is modulated or spread before transmission resulting in a new signal given by

$$
g(t)=\sqrt{\varepsilon} \sum_{i=1}^{N} b(i) s(t-i T)
$$

In which $\varepsilon$ is the signal energy per bit, $T$ is the bit interval and $s(t)$ is a function of limited support, less or

equal to $T$.
In this article we show that if $s(t)=\psi_{\left.0_{\left(2^{j}, 2^{j} k\right.}\right)}(t)_{\text {then the }}$ only wavelet coefficients of $g(t)$ that are different from zero are those of scale $j$. This means that the energy of the new signal is completely concentrated in only one scale. So, if $g(t)$ is used to transmit the information, at the receiving side, we evaluate its wavelet transform, 
and we suppress all coefficients for the scales different from $\mathrm{j}$. These coefficients would be carrying only noise. The recovered signal from the remaining coefficient has its SNR improved by $3 \mathrm{~dB}$.

This technique has been developed to be used in the transmission of CDMA signals of cellular systems employing multiuser detection techniques. It is also useful to improve the signal to interference - SNI - ratio

Equation [2] shows that the DWT for continuous time signals corresponds to a critically sampled version of the CWT defined by (1) in the dyadic scales $s=2^{j}, j=\ldots$, $-1,0,1,2, \ldots$, in which the instants of time in the dyadic scale $s=2^{j}$ are separated by multiples $2^{j}$. The function $\psi_{0}$ of Equation [2] must be defined from a multiresolution analysis - MRA of the signal $x(t)$. Notice that the continuous time MRA theory is similar to that of discrete time.

Figure (1) shows the critical sampling of the time-scale plane by means of the CWT parameters $\left(s=2^{j}\right.$ e $\left.\tau=2^{j} k\right)$ discretization.

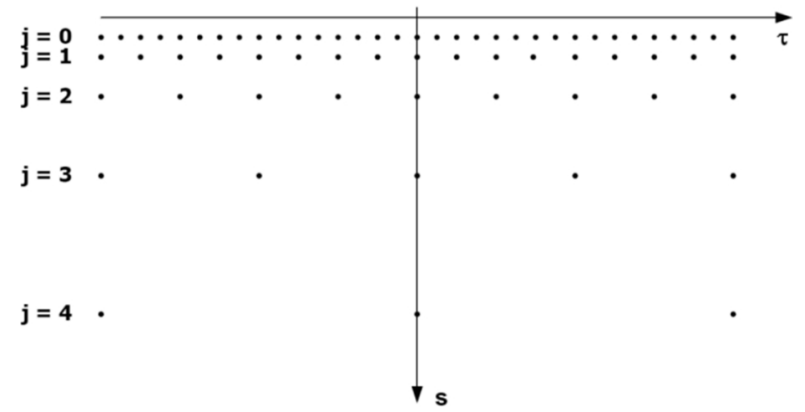

Figure 1. Critical sampling of the time-scale plane by means of the CWT $\left(s=2^{j}\right.$ e $\left.\tau=2^{j} k\right)$ discretization

A MRA is by definition a sequence of closed subspaces $\left\{V_{J}\right\}_{J \in Z}$ de $L^{2}(\mathfrak{R})(5)$ such that:

1)... $V_{2} \subset V_{1} \subset V_{0} \subset V_{-1} \subset V_{-2} \subset \ldots$;

2) $\bigcap_{j \in Z} V_{j}=\{\}$;

3) $\bigcup_{j \in Z} V_{j}=L^{2}(\mathfrak{R})$;

4) $x(t) \in V_{j} \Leftrightarrow x\left(2^{j} t\right) \in V_{0, j}>0$ (in which $\mathrm{t}$ denotes time and $\mathrm{x}(\mathrm{t})$ is an energy signal);

5) There is a function $\phi_{j}(t)=2^{-j / 2} \phi_{0}\left(2^{-j} t\right)$ in $V_{j}$, called scale function, such that the set $\left\{\phi_{i, k}, K \in Z\right\}$ is an orthonormal basis of $V_{j}$, with $\phi_{j, k}(t)=2^{-\mathrm{j} / 2} \phi_{0}\left(2^{-j} t-k\right), \forall J$, $K \in Z$.

The subspace $V_{j}$ is known as the approximation space, associated to the time scale $s_{j}=2^{j}$ (assuming that $V_{0}$ is the approximation space with unit scale.)

If the $x(t)$ projection on $V_{j}$ is represented by the scale coefficients

$$
u_{j, k}=\left\langle\phi_{j, k}, x\right\rangle=\int_{-\infty}^{\infty} 2^{-j / 2} \phi_{0}^{*}\left(2^{-j} t-k\right) x(t) d t,
$$

Then the properties 1 and 3 assume that

$$
\lim _{j \rightarrow-\infty} \sum_{k} \phi_{j, k}(t) u_{j, k}=x(t), \forall x \in L^{2}(\Re) .
$$

Property 4 implies that the subspace $V_{j}$ is a scaled version of subspace $V_{0}$ (multiresolution).

The orthonormal basis mentioned in property 5 is obtained by time shifting the low-pass function $\phi_{j}$.

$$
S_{j}(t)=\sum_{k} \phi_{j, k}(t) u_{j, k} \quad j=\ldots,-1,0,1, \ldots
$$

As $V_{j+I} \subset V_{j}$, we have $S_{j+1}(t)$ is a coarser approximation of $x(t)$ than $S_{j}(t)$.

This fact illustrates the MRA's fundamental idea, that consists of examining the loss of information when one goes from $S_{j}(t)$ to $S_{j+1}(t)$ :

$$
S_{j}(t)=S_{j+1}(t)+\Delta x_{j+1}(t) .
$$

$\Delta x_{j+1}$ (called the detail of $\left.x_{j}(\mathrm{t})\right)$ belongs to the subspace $W_{j+l}$, named detail space that is associated to the fluctuations (or variations) of the signal in the more refined time scale and that corresponds to the orthogonal complement of $V_{j+1}$ in $V_{j}$.

The MRA shows that the detail signals $\Delta x_{j+1}(t)=D_{j+l}(t)$ may be directly obtained by successive projections of the original signal $x(t)$ on wavelet subspaces $W j$.

Moreover, the MRA theory shows that it exists a function $\Psi_{0}(t)$, called "mother wavelet", that is obtained from $\phi_{0}(t)$, and from which a scaled by $j$, and shifted by $k$, version can be obtained as $\Psi_{j, k}(t)=2^{-\mathrm{j} / 2} \phi_{0}\left(2^{-j} t-k\right), k \in$ $Z$ is an orthonormal basis of $W_{j}{ }^{j}$ The detail $D_{j+1}$ is obtained from the equation

$$
D_{j+1}(t)=\sum_{k} \psi_{j+1, k}(t)\left\langle\psi_{j+1, k}(t), x(t)\right\rangle .
$$

The inner-product

$$
\left\langle\psi_{j+1, k}(t), x(t)\right\rangle=\omega_{j+1, k}
$$

denotes the wavelet coefficient associated to scale $j+1$ and discrete time $k$ and $\left\{\psi_{j+1, k}(t)\right\}$ is a family of wavelet functions that generates the subspace $W_{j+1}$, orthogonal to subspace $V_{j+1}\left(W_{j+1} \perp V_{j+1}\right)$, i.e., 


$$
\left\langle\psi_{j+1, n}, \phi_{j+1, p}\right\rangle=0, \quad \forall n, p
$$

Therefore, the detail signal $D_{j+1}$ belongs to the complementary subspace $W_{j+1}$ of $V_{j}$, because

$$
V_{j}=V_{j+1} \oplus W_{j+1} .
$$

That is, $V_{j}$ is given by the direct addition of $V_{j+1}$ and $W_{j+1}$ and this means that any element in $V_{j}$ may be determined from the addition of two orthogonal elements belonging to $V_{j+1}$ and $W_{j+1}$ (6) Iterating [9], we have

$$
V_{j}=W_{j+1} \oplus W_{j+2} \oplus \cdots
$$

\section{How the noise can be reduced by means of a wavelet modulation}

As previously commented in the "Summary", one of the possible techniques to reduce the noise at the receiving side of a communications system employs the wavelet transform (7). The typical noise-reduction method based on wavelet transform is called denoising, which is used to reduce noise in images for instance. Comparing this typical denoising to the method presented here, the main difference to be noted is that here one has access to the source of the signal, so it is possible to process the signal conveniently, in a way that when it arrives at the receiver, that initial processing of the transmission can be adequately explored, so this new denoising or modulation can be made more effectively. As shown in Section II, the wavelet transform is a representation in the plane time-frequency. Any signal can be completed recovered by means of the wavelet coefficients and the inverse wavelet transform. An approximation of the signal can be obtained using a subset of the wavelet coefficients.

If, for example, the received signal has a reasonable SNR but not good enough to achieve the desired BER, the elimination of the wavelet coefficients that are less than a given threshold may result in an improved SNR because the small coefficients carry more information about the noise than the desired signal. On the other hand, there are certain signals that have the energy concentrated in a part of the spectrum. So, if only the wavelet coefficients corresponding to this part of the spectrum are used, the SNR also improves. However, in general, in digital transmission systems, none of abovementioned conditions prevails.

\section{RESULTS AND DISCUSSION}

In this section we will show by means of simulation results that the conclusions concerning the de-noising can be verified. The simulations were performed using the software known as "R" (8).

We started by generating an unit power random sequence $\{b(m)\}=\{\mathrm{b}(1), \mathrm{b}(2), \ldots, \mathrm{b}(\mathrm{N})\}$ where $N=$ 256. In Fig. 2, the top plot is the generated sequence. The remaining plots are the wavelet coefficients for different scales from $j=1$ up to $j=4$. The horizontal axis is time. The actual values of the wavelet coefficients are not important for the purposes of this article; the objective here is to realize that in all scales they are different from zero. This is also true for the coefficients that are not shown in the figure.

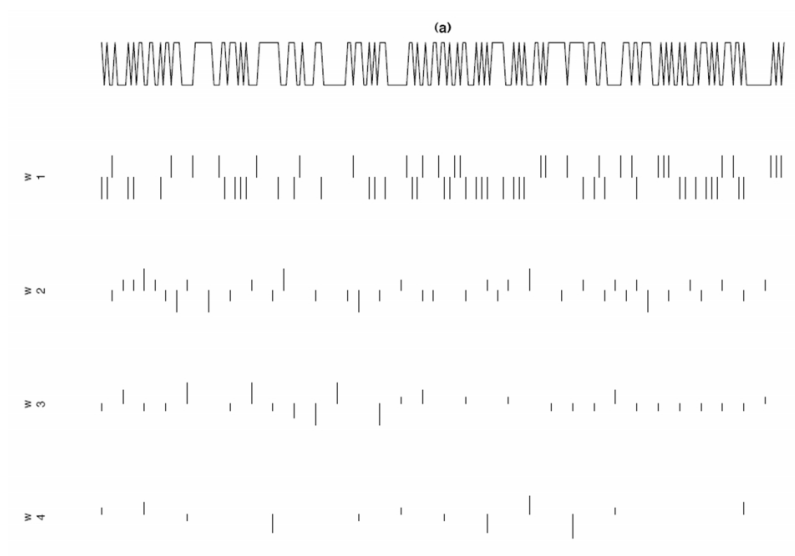

Figure 2. A 256-point random sequence and its associated wavelet coefficients up to scale $j=4$.

Fig. 3 shows the wavelet spectrum corresponding to the sequence presented in Fig. 2. The wavelet variance on the vertical axis is a measure of the energy of the signal, and it is obtained by squaring the wavelet coefficients and summing them up; on the horizontal each number corresponds to the scales of the coefficients. It can be seen that for all practical purposes, it can be considered flat as its variation is below $1 \mathrm{~dB}$. This is a spectrum of white noise, and the signal does not have any interesting characteristic to be exploited by any denoising technique. 


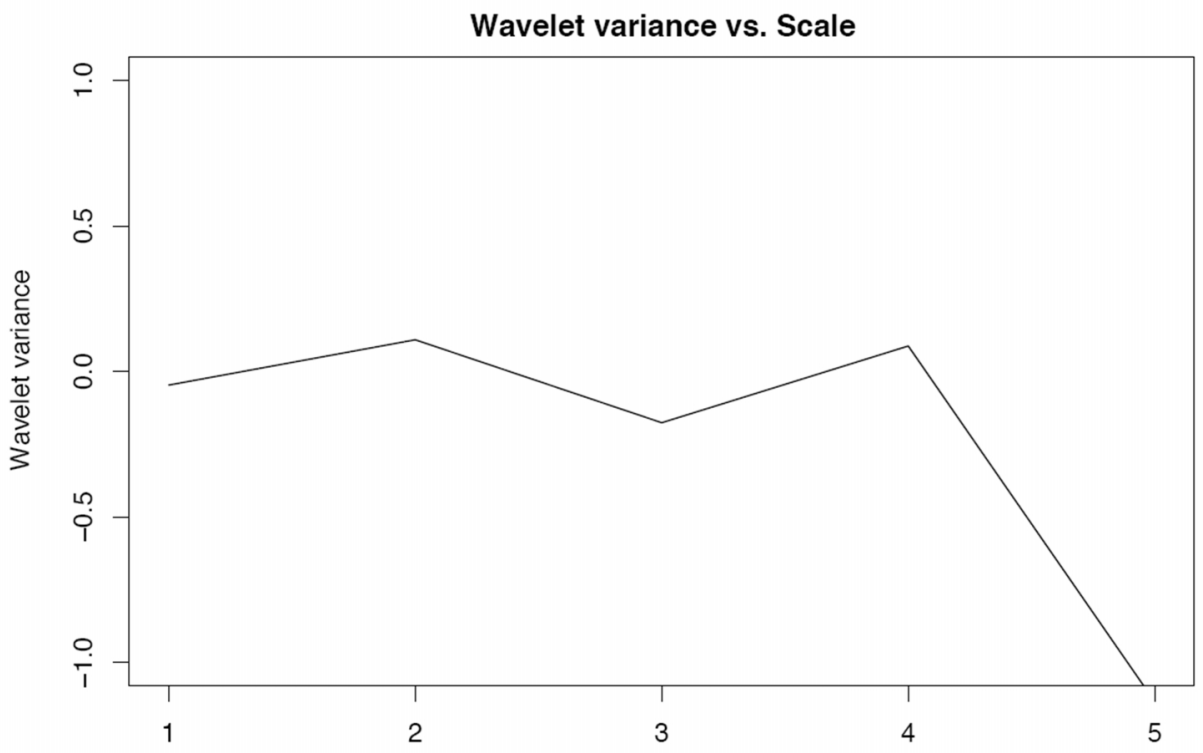

Figure 3. Wavelet spectrum of a 256-point random sequence.

Then we implemented the wavelet modulation technique as given by [3]. The conclusions arrived in Section III do not depend on any particular wavelet function. They are the same for Haar, Daubechies, Coiflet, etc. For the sake of simplicity, we adopted the Haar function. As this function assumes only values \pm 1 , it can be implemented without any approximation error. So, each symbol of the sequence $b(m)=b(1), b(2), \ldots, b(N)$ has been multiplied by the vector haar $1=\{-1,1,-1,1\}$. The new sequence has 1024 points, and this corresponds to a four times spread in frequency. The new sequence and its wavelet coefficients up to scale $j=4$ are shown in Fig. 4. The top row is new sequence. Due to the number of points the individual symbols are not distinguishable. The next row represents the wavelet coefficients at scale $j=1$. It can be seen that they are not equal to zero. From scale $j=2$ all wavelet coefficients are equal to zero, according to [12]:

$$
\begin{aligned}
& g(t)=\sqrt{\varepsilon} \sum_{i=1}^{N} b(i) \psi_{0_{\left(2^{j}, 2^{j} k\right)}}(t-i T) \\
& W_{\psi}(s, \tau)=\left\langle\psi_{\left.0_{(s, \tau)}, g(t)\right\rangle}\right. \\
& W_{\psi}(s, \tau)=\left\langle\psi_{0_{(s, \tau)},}, \sqrt{\varepsilon} \sum_{i=1}^{N} b(i) \psi_{0_{\left(2^{j}, 2^{j} k\right)}}(t-i T)\right\rangle \\
& W_{\psi}(s, \tau)=\sqrt{\varepsilon} \sum_{i=1}^{N} b(i)\left\langle\psi_{\left.0_{(s, \tau)}, \psi_{0_{\left(2^{j}, 2^{j} k\right)}}(t-i T)\right\rangle}\right.
\end{aligned}
$$

If during the transmission of the wavelet modulated sequence it is corrupted by noise, then its wavelet coefficients from scale $j=2$ on will not be identically equal to zero. However, any wavelet coefficient different from zero represents a portion of the noise energy, and it can be eliminated from the signal representation without distorting the original sequence.

Wavelet decomposition of a coded random sequence

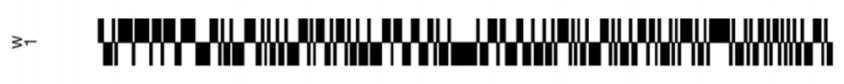

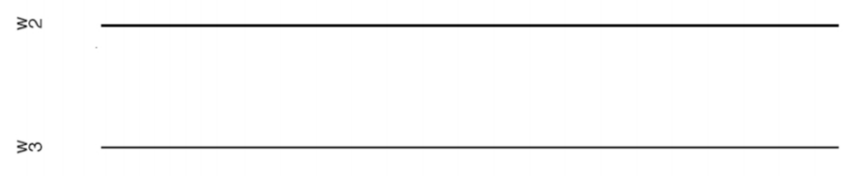

Figura 4. Wavelet modulated sequence and its associated wavelet coefficients.

Wavelet decomposition is also power decomposition, i.e.,

$$
P_{j}=\sum_{k=1}^{N / 2^{j}} \omega_{j, k}^{2}
$$

is the signal power in the $\left(\frac{1}{2^{j+1}}, \frac{1}{2^{j}}\right)$ fre quency 
range. It is important to note that we employ normalized frequencies, and the entire range is $(0,1 / 2)$ It is also known that the Additive White Gaussian Noise - AWGN - has a flat spectrum, i.e., its power is uniformly distributed across the frequency range. So, the noise power in a given frequency range is proportional to the frequency range length. As frequency range covered by the wavelet coefficients in scales from $j=2$ on is $(0,1 / 4)$ i.e., half of the whole frequency, then the noise power in this range is half of the total noise power. Therefore, eliminating the wavelet coefficients in such scales means the elimination of half of noise power of the received signal and corresponding increase of $3 \mathrm{~dB}$ of the SNR at the detection point
The wavelet spectrum corresponding to the sequence modulated by the haar1 function has only one value different from zero for $j=1$. As it has been shown before, the haar1 function modulation produces wavelet coefficients different from zero only for $j=1$ and this directly reflected on the wavelet spectrum. On the other hand, the haar2 function is a first dilation of haar1 and produces signals that belong to the subspace $W_{2}$. Accordingly, their wavelet coefficients that are different from zero are those corresponding to $j=2$. The wavelet spectrum presents also only one value different from zero for $j=2$. The same reasoning is valid for the haar3 function, and its wavelet spectrum has only one value different from zero for $j=3$.

Table 1. Three different Haar functions

\begin{tabular}{|l|cccc|ccccc|ccccc|ccc|}
\hline Vector & \multicolumn{11}{|c|}{ Component } \\
\hline haar1 & -1 & 1 & -1 & 1 & -1 & 1 & -1 & 1 & -1 & 1 & -1 & 1 & -1 & 1 & -1 & 1 \\
haar2 & -1 & -1 & 1 & 1 & -1 & -1 & 1 & 1 & -1 & -1 & 1 & 1 & -1 & -1 & 1 & 1 \\
haar3 & -1 & -1 & -1 & -1 & 1 & 1 & 1 & 1 & -1 & -1 & -1 & -1 & 1 & 1 & 1 & 1 \\
\hline
\end{tabular}

Consider now three different Haar functions given This result has very important implications that can by the vectors shown in Table I. The vector haar1 represents the "mother wavelet" function, haar2, and haar3; the first two dilations of haar1. Each of these vectors has been used to wavelet modulate a random sequence and the wavelet coefficients for each of them have been evaluated. The wavelet spectra for the three sequences are shown in Fig. 5.

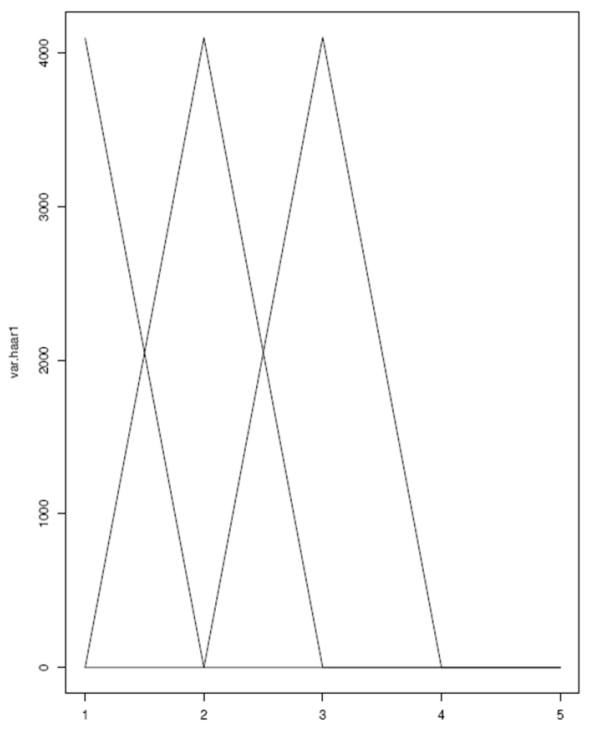

be summarized as follows:

- within certain limits dictated by practical considerations, we can freely choose the Haar function to be used as the modulating signal;

- for any Haar function that is selected only the wavelet coefficients of only one specific scale are different from zero;

- at the receiving side of the communication system, the noise power can be reduced by zeroing the wavelet coefficients of those scales that are not selected by the chosen Haar function;

- the noise power reduction provided by this procedure is at least $3 \mathrm{~dB}$.

If, for example, we choose to use the function haar2, the wavelet coefficients which are different from zero correspond to $j=2$, and they cover the frequency range $\left(1 / 2^{3}, 1 / 2^{2}\right)$ The length of the frequency range is $1 / 2^{3}$. The noise power reduction that can be achieved is

$$
\frac{\frac{1}{2}-\frac{1}{2^{3}}}{\frac{1}{2}}=\frac{3}{4} \text { or } 6 \mathrm{~dB} \text {. }
$$

Figure 5. Wavelet spectra of three sequences obtained by wavelet modulation using Haar mother function and its two first dilations. 
In general terms, if we choose to wavelet modulate a sequence with a Haar function that generates a new signal which belongs to subspace $W_{j}$. The achievable noise power reduction will be $j \mathrm{x} 3 d B$.

In DS-CDMA systems each user has a specific code, and this code allows the receiver to separate the information of interest from the interference produced by other users that transmit simultaneously. It is important to notice that in a real system the wavelet modulation technique would be used in association with the spreading technique and not as a substitution for it. In fact, the signal to be transmitted would be

$$
g(t)=\sqrt{\varepsilon} \sum_{i=1}^{N} b(i) s(t-i T) \psi_{0_{\left(2^{j}, 2^{j} k\right)}}(t-i T),
$$

However, for the sake of simplicity we have not considered the spread signal in this article, and because the conclusions would be exactly the same.

Nevertheless, as shown in [14], if we wavelet modulate the sequences of different users using different Haar functions, the wavelet coefficients that are different from zero will be in different scales for different users. If, for example, for user 1 we use the haar1 function, the wavelet coefficients different from zero will be scale $j=1$. If at the receiving side we eliminate all wavelet coefficients for $j=2$ on, we will eliminate half of the noise and power and all interference power coming from users that have their sequences modulated by Haar functions other than the haar1

Therefore, the wavelet modulation increases both SNR and SIR ratios.

\section{CONCLUSIONS}

After a brief introduction of the wavelet transform in which we have shown how any signal can be represented by means of the wavelet coefficients, and how it can be recovered using wavelet transform inverse, we introduced the concept of wavelet modulation.

The wavelet modulation is a kind of spread spectrum technique that uses wavelet functions as the spreading signals. It has been shown that due to the orthonormality property of the one scale determined by the chosen wavelet function.

Such property can be used to implement a denoising technique, and the noise power reduction is at least $3 \mathrm{~dB}$.

The wavelet modulation can also be used to discriminate among different users and improve the SNI ratio as well.

\section{ACKNOWLEDGMENTS}

We want to express our gratitude to Dr. Alexandre Lima for the productive discussions during the development of this work and recognize that most of wavelet review section is based on the material available in his $\mathrm{PhD}$ thesis. 


\section{BIBLIOGRAPHY}

1. Verdu, S. Multiuser Detection. Cambridge University Press. 2005.

2. Coifman, R., Meyer, Y., and Wickerhauser. "Wavelet analysis and signal processing," in Wavelets and their applications. Boston: Jones and Bartlett, 1992.

3. Daubechies, L. Ten Lectures on Wavelets. Philadelphia: SIAM. 1992.

4. Percival, D.B. and Walden, A.T. Wavelet Methods for Time Series Analysis. Cambridge University Press. 2000.

5. Mallat, S.G. "Multiresolution approximations and wavelet orthonormal bases of ." Transactions of the American Mathematical Society, 1989; 315, 69-87.

6. Daubechies, I. "Orthonormal bases of compactly supported wavelets." Comm. Pure Appl. Math. 1988; 41, 909-996.

7. Momen, A., Ahmadi-Noubari, H., and Mirzaee, A. "A new de-noising method in spread spectrum systems based on wavelet transform." IEEE Canadian Conference on Electrical and Computer Engineering, 2006; 1, 2127-2130.

8. Jones, O., Maillardet, R., and Robinson, A. Introduction to Scientific Programming and Simulation Using R. Chapman and Hall/CRC. 2009. 